\title{
Structural Fingerprinting of Nanocrystals in the Transmission Electron Microscope Supported by Open-Access Crystallographic Databases
}

\author{
Peter Moeck and Sergei Rouvimov
}

Nano-Crystallography Group, Department of Physics, Portland State University, Portland, OR 97207-0751 \& Oregon Nanoscience and Microtechnologies Institute

It is well known that nanocrystals cannot be fingerprinted structurally from powder X-ray diffraction patterns [1-3]. Three strategies for the structurally identification of nanocrystals in a Transmission Electron Microscope (TEM) have, therefore, been developed. Either a single High-Resolution Transmission Electron Microscopy (HRTEM) image or a single Precession Electron Diffractogram (PED) can be employed. The structural identification information is in both cases collected from an individual nanocrystal. PED from fine-grained crystal powders may also be utilized.

The structural information that can be extracted from a HRTEM image of an approximately $5 \mathrm{~nm}$ thick nanocrystal is the projected reciprocal lattice geometry, the plane symmetry group, and a few structure factor amplitudes and phase angles. While the structure factor amplitudes suffer from dynamical diffraction effects and are in addition modified by the (not precisely known) contrasttransfer function of the objective lens, the structure factor phase angles are remarkably stable against dynamical diffraction effects and slight crystal misorientations [3,4].

Except for the structure factor phase angles, the same "kind" of structural information can be extracted from a PED pattern. Instead of the plane symmetry of the HRTEM images, the 2D point symmetry of the PED is used and no structure factor phases are accessible. The information that can be utilized for structural fingerprinting is in the PED case, however, not limited to the directly interpretable resolution of the TEM. Since precession electron diffraction avoids crystal orientations that result in the simultaneous excitation of more than one strong diffracted beam (as much as this is possible), quasi-kinematic reflection intensities are obtained for nanocrystals with thicknesses up to approximately $25 \mathrm{~nm}$. Simultaneously present reflections in higher order Laue zones and systematic absences in both the higher and the zero order Laue zones of a few projections allow frequently for an unambiguous determination of the space group [1-3].

Searching for structural information that is extracted from HRTEM images or PEDs in comprehensive databases and matching it with high figures of merit to that of candidate structures shall allow for highly discriminatory identifications of nanocrystals, even without additional chemical information as obtainable in analytical TEMs. As an alternative to the well known commercial databases, one may use open-access databases such as the Crystallography Open Database (COD) which provides more than 130,000 crystal structure data sets [5,6]. The COD's mainly inorganic and educational subsets and the Nano-Crystallography Database, see Fig. 1 (ref. [7]), may also be used [8].

\section{References}

[1] P. Moeck and S. Rouvimov, Zeits. Kristallogr. 225 (2010) 110.

[2] P. Moeck and S. Rouvimov, in: Drug Delivery Nanoparticles: Formulation and Characterization, Y. Pathak and D. Thassu (eds.), Informa, 2009 (Drugs and the 
Pharmaceutical Sciences, Vol. 191), New York, pp. 270-313.

[3] P. Moeck and P. Fraundorf, Zeits. Kristallogr. 222 (2007) 634, expanded version in open access: arXiv:0706.2021.

[4] R. Bjorge, MSc thesis, Portland State University, May 9, 2007; Journal of Dissertation Vol. 1 (2007), open access: http://www.scientificjournals.org/journals 2007/j_of_dissertation.htm

[5] http://cod.ibt.lt mirrored at http://nanocrystallography.org and http://www.crystallography.net and http://cod.ensicaen.fr

[6] S. Gražulis, D. Chateigner, R. T. Downs, A. F. T. Yokochi, M. Quirós, L. Lutterotti, E. Manakova, J. Butkus, P. Moeck, and A. Le Bail, J. Appl. Cryst. 42 (2009) J. Appl. Cryst. 42 (2009) 726, open access: http://journals.iucr.org/j/issues/2009/04/00/kk5039/kk5039.pdf.

[7] http://nanocrystallography.research.pdx.edu/CIF-searchable

[8] This research was supported by both the Oregon Nanoscience and Microtechnologies Institute and the Army Research Laboratories.

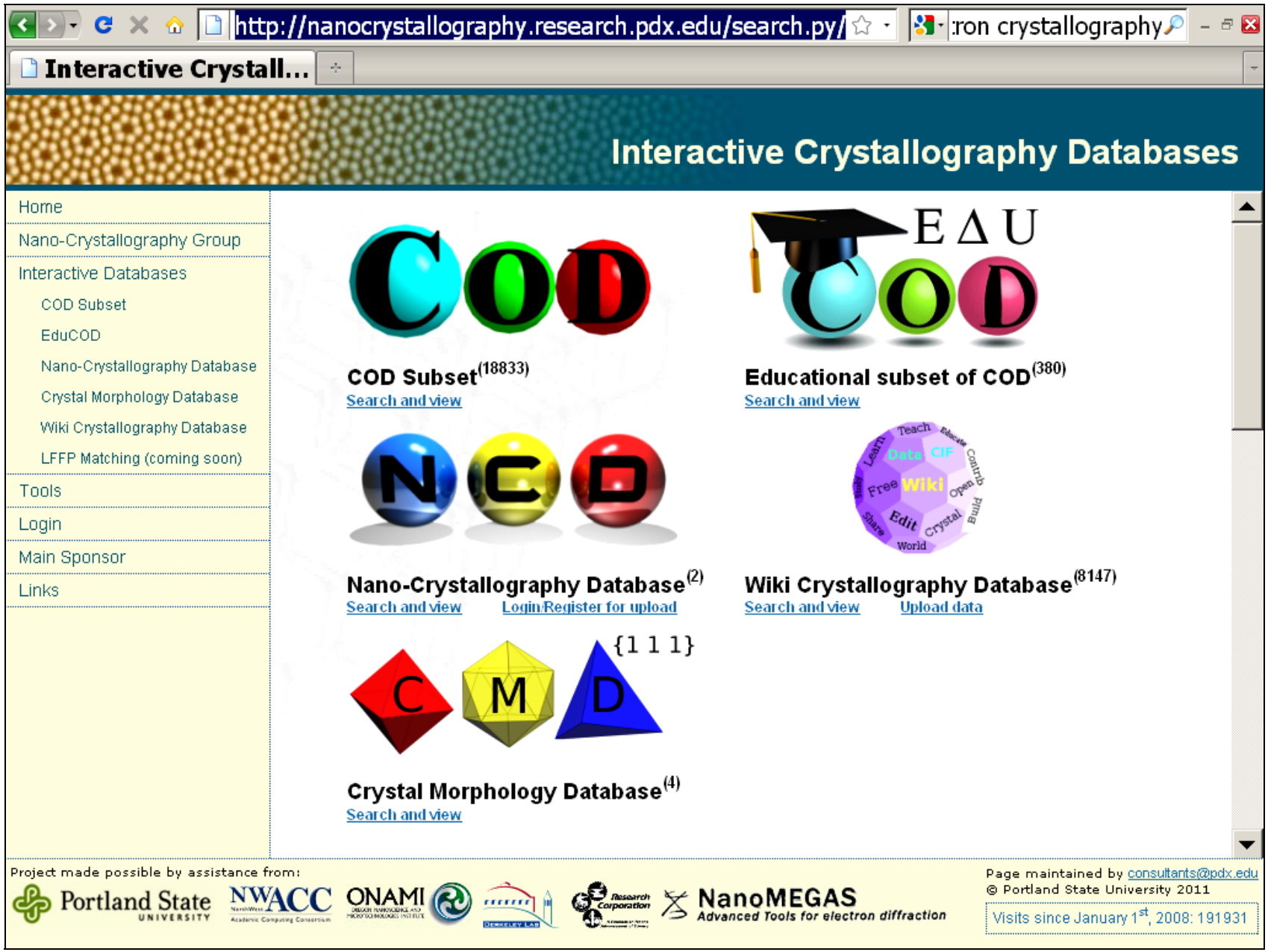

FIG 1. Screenshot of http://nanocrystallography.research.pdx.edu/CIF-searchable, which provides open access to some 20,000 comprehensive crystallographic datasets. 